\title{
Características Antropométricas do Palato Ósseo de uma Amostra Brasileira de Esqueletos Identificados
}

\section{Anthropometric Characteristics of the Bony Palate in a Brazilian Sample of Identified Skeletons}

\author{
Larissa Chaves Cardoso Fernandes ${ }^{1}$ \\ Patrícia Moreira Rabello² \\ Eduardo Daruge Júnior ${ }^{3}$ \\ Gabriela Granja Porto ${ }^{4}$ \\ Marcus Vitor Diniz de Carvalho ${ }^{5}$ \\ Evelyne Pessoa Soriano 4,6
}

\section{RESUMO}

Objetivo: Este estudo teve como objetivo descrever as características antropométricas do palato ósseo de uma amostra brasileira de esqueletos identificados Metodologia: Foi desenvolvido um estudo de corte transversal, por meio da análise de 178 crânios secos humanos, oriundos do Arquivo de Ossadas do Departamento de Odontologia Social da Faculdade de Odontologia de Piracicaba (FOP/UNICAMP), São Paulo. Utilizando paquímetro digital, foram realizadas medições lineares que incluíam forame incisivo à espinha nasal posterior (FI-ENP), forame palatino maior direito ao forame palatino maior esquerdo (FPMD-FPME), forame incisivo ao forame palatino maior direito (FI-FPMD) e forame incisivo ao forame palatino maior esquerdo (FI-FPME). Os testes t-Student, F(ANOVA), Tukey, LSD, Kruskal Wallis e Pearson, com nível de significância de $5,0 \%$. Resultados: Do total de crânios analisados, $54,5 \%$ eram do sexo masculino, com mediana de idade de 56 anos. Todas as medidas analisadas apresentaram dimorfismo sexual, onde homens possuíram maiores dimensões do que o sexo oposto $(p<0,001)$. Do total de palatos estudados, 104 eram pertencentes a indivíduos brancos, 49 a miscigenados e 25 a negros. As medidas FI-ENP, FPMD-FPME e FI-FPME apresentaram-se maiores em negros, seguidos dos miscigenados e brancos. Conclusão: Conclui-se que as características do palato ósseo podem ser aplicadas como maior confiabilidade para a estimativa do sexo na amostra brasileira estudada.

\section{DESCRITORES}

Palato, Antropologia Forense. Odontologia Legal. Crânio. Dimorfismo Sexual. Grupos de Populações Continentais.

\begin{abstract}
Objective: This study aimed to describe the anthropometric characteristics of the bony palate in a Brazilian sample of identified skeletons. Methodology: This was a cross-sectional study of 178 human skulls from the Laboratory of Forensic Physical Anthropology at Piracicaba Dental School, University of Campinas, São Paulo. A digital pachymeter was used to perform linear measurements from the incisive foramen to the posterior nasal spine (IF-PNS); right greater palatine foramen to left greater palatine foramen (RGPF-LGPF); incisive foramen to the right greater palatine foramen (IF-RGPF); and incisive foramen to the left greater palatine foramen (IF-LMPF). The data were submitted to Student's $t$, F (ANOVA), Tukey, LSD, Kruskal Wallis and Pearson tests, with a significance level of $5.0 \%$. Results: Of the total skulls analyzed, $54.5 \%$ were from males, with a median age of 56 years. All the study measures presented sexual dimorphism, in which males had larger dimensions than females $(p<0.001)$. Of the total palates studied, 104 were from white individuals, 49 were from mixed-race and 25 from black individuals. The IF-PNS, RGPF-LGPF and IF-LGPF measurements were greater in black individuals, followed by mixed-race and white individuals. Conclusion: In conclusion, the characteristics of the bony palate can be applied with higher reliability to estimate sex in Brazilian sample studied.
\end{abstract}

\section{DESCRIPTORS}

Palate. Forensic Anthropology. Forensic Dentistry. Skull. Sexual Dimorphism. Continental Population Groups.

${ }^{1}$ Cirurgiã-Dentista, Mestra, Doutoranda em Biologia Buco-Dental, Área de Anatomia, Departamento de Odontologia Social, Faculdade de Odontologia de Piracicaba/Universidade Estadual de Campinas - FOP/UNICAMP, Piracicaba - São Paulo, Brasil.

${ }^{2}$ Cirurgiã-Dentista, Mestra, Doutora, Universidade Federal da Paraíba - UPFB, Departamento de Clínica e Odontologia Social, Área de Odontologia Legal, João Pessoa - Paraíba, Brasil.

${ }^{3}$ Cirurgião-Dentista, Mestre, Doutor, Departamento de Odontologia Social, Faculdade de Odontologia de Piracicaba/Universidade Estadual de Campinas - FOP/UNICAMP, Piracicaba - São Paulo, Brasil.

${ }^{4}$ Cirurgiã-Dentista, Mestra, Doutora, Mestrado em Perícias Forenses, Faculdade de Odontologia de Pernambuco/Universidade de Pernambuco - FOP/UPE, Camaragibe - Pernambuco, Brasil.

${ }^{5}$ Médico, Mestre, Doutor, Mestrado em Perícias Forenses, Faculdade de Odontologia de Pernambuco/Universidade de Pernambuco FOP/UPE, Camaragibe - Pernambuco, Brasil.

${ }^{6}$ Perita do Departamento de Medicina e Odontologia Legal, Instituto de Polícia Científica, João Pessoa - Paraíba, Brasil. 


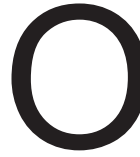
processo de identificação de vítimas mortais é uma necessidade social, jurídica e humanitária ${ }^{1,2}$. A Odontologia

Legal, ramo da Medicina Forense, colabora com a Justiça na realização de exames nos arcos dentais e estruturas correlatas ${ }^{3}$ de cadáveres, restos mortais carbonizados, despedaçados, em adiantado estado de decomposição ou esqueletizados ${ }^{2,4}$. Com a aplicação de técnicas e metodologias forenses no crânio, sejam as mesmas de cunho métrico e morfológico, é possível chegar a informações quanto ao sexo, a espécie, a afinidade populacional, a idade no momento do óbito e a estatura da ossada questionada ${ }^{2}$.

A adoção de protocolos forenses que levem a comprovação da identidade humana por meio de estudos das características cranianas tem sua importância no fato de que, frequentemente, é esta estrutura óssea o único subsídio legal encontrado na cena arqueológica, durante a investigação criminal e/ ou no desastre em massa ${ }^{1}$. Tal fato é explicado uma vez que a conformação e o elevado peso do crânio articulado, em comparação com outros ossos do corpo humano, dificultam o transporte do mesmo por animais ou outras forças naturais.

Dentre as estruturas cranianas de interesse, o palato ósseo, que compreende o limite superior da cavidade bucal e é constituído pela face inferior dos processos palatinos da maxila juntamente às faces inferiores das lâminas horizontais dos ossos palatinos ${ }^{5-7}$, possui acidentes anatômicos que resistem aos mais diversos processos transformativos que acometem o corpo humano após a morte. As diferenças na morfometria do palato duro parecem ser úteis na indicação de pilares do protocolo antropológico - sexo, ancestralidade e estimativa de idade ${ }^{8,9}$.

Partindo do princípio de que a con- fiabilidade de um laudo de identificação humana baseia-se no somatório de elementos periciados $^{8,10}$, não havendo método forense de estabelecimento da identidade que se sobreponha a outro ${ }^{5,8,11-13}$, e que existem diferenças métricas e morfológicas no estudo de peças ósseas oriundas de populações distintas ${ }^{14}$, este estudo teve como objetivo avaliar as características antropométricas do palato ósseo, relacionando-as com o sexo e a ancestralidade, em uma amostra do sudeste brasileiro.

\section{METODOLOGIA}

Este estudo de corte transversal teve um universo composto por 192 ossadas, previamente catalogadas quanto ao sexo, a ancestralidade (de acordo com a certidão de nascimento) e a idade à morte, pertencentes ao Arquivo de Ossadas do Departamento de Odontologia Social, área da Odontologia Legal, da Faculdade de Odontologia de Piracicaba/Universidade Estadual de Campinas (FOP/UNICAMP). Após a exclusão dos crânios com malformações, anomalias severas, maxila seccionada sagitalmente, com extensa perda de dentes e/ou edêntula, além daqueles que apresentavam torus palatino, traumas aparentes ou patologias ósseas graves que comprometiam a integridade do palato ósseo, a amostra resumiu-se a 178 crânios secos.

Como variáveis do estudo, foram considerados: o sexo (masculino e feminino) e a ancestralidade (branca, miscigenada e negra).

No palato ósseo foram analisados os acidentes anatômicos correspondentes ao forame incisivo (FI), à espinha nasal posterior (ENP) e aos forames palatinos maiores direito (FPMD) e esquerdo (FPME) (Figura 1).

Durante toda a coleta dos dados, foram utilizados pelos pesquisadores luvas, máscaras, gorro e avental descartável, uma 
Figura 1. Vista do palato ósseo com os pontos craniométricos de interesse.

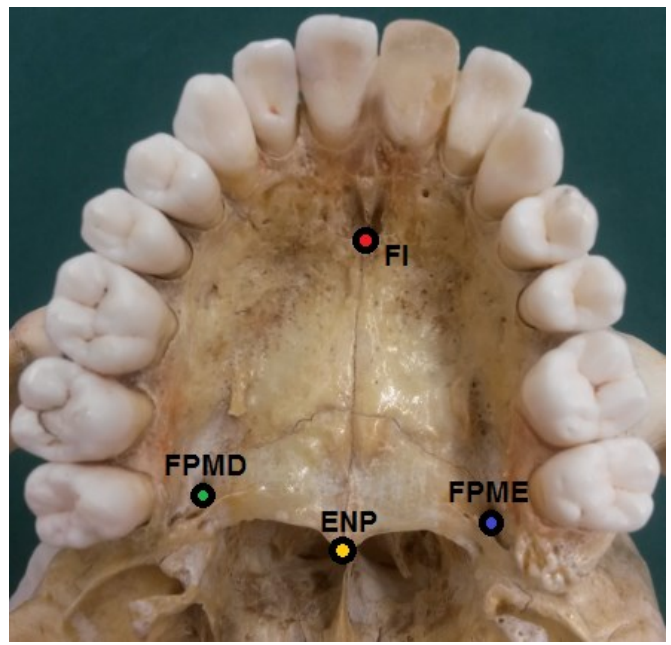

Fonte: Arquivo da pesquisa.

vez que os materiais coletados na pesquisa eram originados de ossadas humanas, procurando-se, dessa forma, evitar qualquer possibilidade de contaminação com algum micro-organismo eventualmente presente nas superfícies ósseas examinadas.

Foi utilizado um paquímetro digital (Stainless-Hardened ${ }^{\circledR}-150$ mm, Mauá, São Paulo, Brazil) para a obtenção das medidas lineares: distância entre o forame incisivo e o forame palatino maior direito (FI $\leftrightarrow$ FPMD), distância entre o forame incisivo e o forame palatino maior esquerdo (FI $\leftrightarrow$ FPME), distância entre o forame palatino maior direito $\mathrm{e}$ o forame palatino maior esquerdo (FPMD $\leftrightarrow$ FPME) e distância entre o forame incisivo e a espinha nasal posterior (FI $\leftrightarrow$ ENP) (Figura 2 - A, B, C e D).

Um estudo piloto, por meio da medição de 25 crânios secos do acervo supracitado, foi aplicado previamente, com o intuito de treinar a examinadora, sendo o intervalo de tempo entre a primeira e segunda análises de sete dias. Através da estatística Kappa, a concordância entre os exames foi interpretada como ótima $(\geq 0,92)$, indicando criterioso processo métrico. Por não ter sido observada a necessidade de ajustes no estudo piloto, todos os crânios medidos nesta etapa foram incluídos na amostra final.

É importante destacar que, devido à alta sensibilidade do instrumento, após cada medida, as pontas do mesmo eram unidas e o calibrador zerado, evitando pequenas possíveis variações do equipamento que poderiam alterar ou prejudicar o resultado do estudo.

Os dados obtidos foram processados por meio do programa estatístico Statistical Package for Social Sciences, versão 23.0 (SPSS Inc., Chicago, IL, EUA), com o qual utilizaram-se técnicas de estatística descritiva e inferencial através dos testes t-Student com variâncias iguais, F(ANOVA), Tukey, LSD, Kruskal Wallis e Pearson. Considerou-se o nível de significância de $5 \%(p \leq 0,05)$.

Esta pesquisa segui as determinações da Resolução n 466/12, do Conselho Nacional de Saúde, Ministério da Saúde, sendo o 
Figura 2. Vista do palato ósseo com as medidas lineares de interesse: A - Distância FI $\leftrightarrow$ FPMD; B - Distância FI $\leftrightarrow$ FPME; C - Distância FPMD $\leftrightarrow$ FPME e D - Distância FI $\leftrightarrow$ ENP.
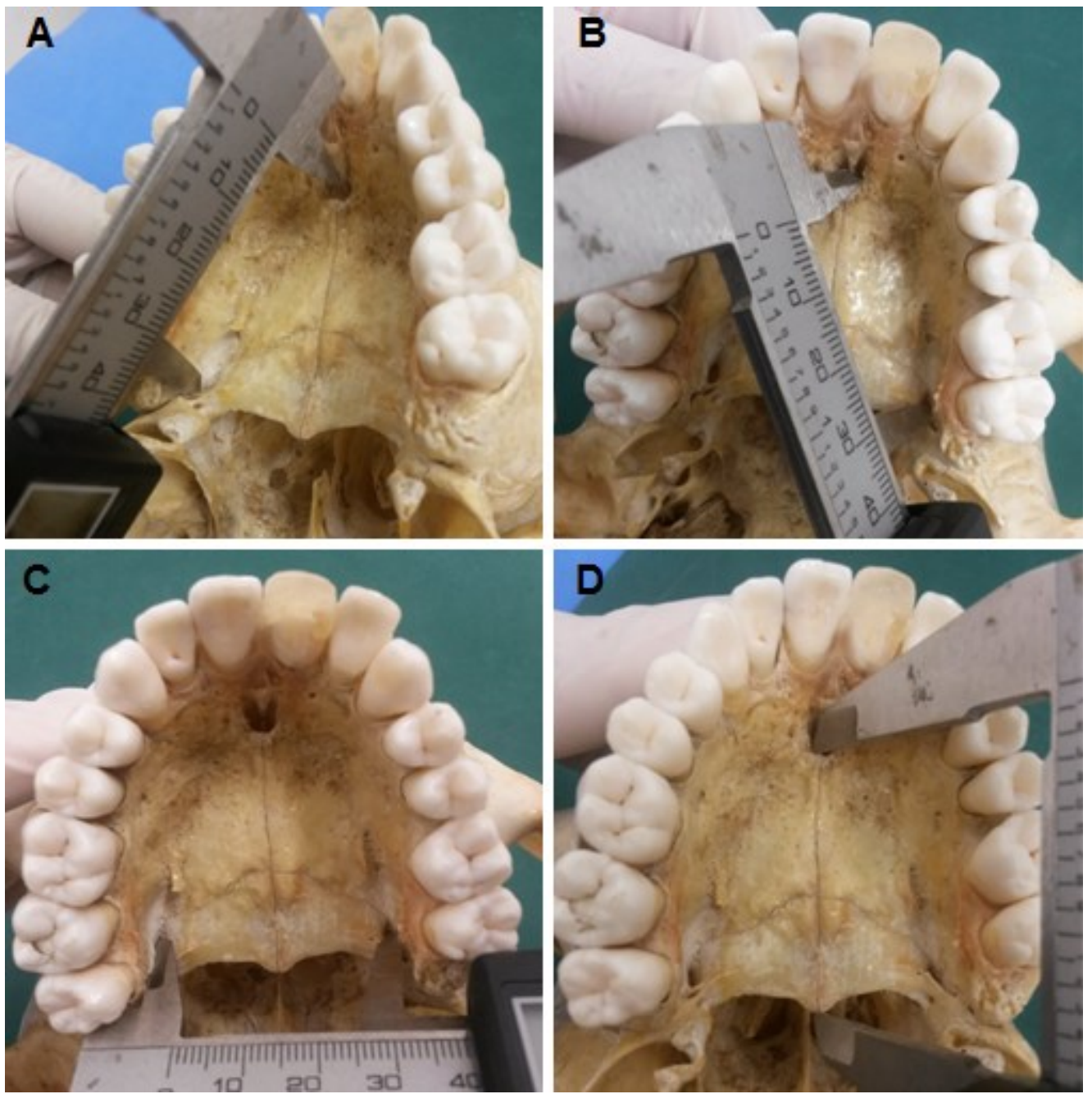

Fonte: Arquivo da pesquisa.

devido projeto submetido e aprovado pelo Comitê de Ética em Pesquisa do Centro de Ciências da Saúde da Universidade Federal da Paraíba (CAAE: 45781415.0.1001.5188).

\section{RESULTADOS}

No total foram analisados 178 crânios secos. $54,5 \%$ da amostra era do sexo masculino e $45,5 \%$ do sexo feminino, sendo a média e mediana da idade de 55,87 anos e 56,00 anos, respectivamente, com desvio padrão de 20,21 anos, o que mostra uma variabilidade não elevada uma vez que a referida medida foi inferior à metade da média correspondente. Quanto à idade, a maior parte da amostra $(46,1 \%)$ correspondeu a crânios secos que apresentavam 60 anos ou mais no momento da morte, seguido dos que tinham entre 40 e 59 anos (32,6\%) e até 39 anos (21,3\%). A 
maioria $(58,4 \%)$ possuía ancestralidade branca, seguida dos miscigenados $(27,5 \%)$ e dos negros $(14,0 \%)$.

A Tabela 1 destaca que a variabilidade expressa pelo desvio padrão (DP) mostrou-se reduzida, uma vez que a referida medida foi inferior a 1/5 das médias correspondentes.

Quanto ao sexo, todas as variáveis analisadas mostraram médias correspondentemente mais elevadas no sexo masculino do que no sexo feminino, sendo tais diferenças estatisticamente significativas $(p<0,001)$ (Tabela 2).

No estudo que relacionou as faixas etárias com as variáveis da região do palato (Tabela 3), nenhuma das medidas mostrou-se significativa $(p>0,05)$ para a margem de erro fixada.

No que tange à ancestralidade, apenas as dimensões FI-FPMD e FI-FPME demonstraram diferenças significativas. Constatou-se, ainda, que a variável FI-FDMP apresentou-se menos elevada entre os brancos e mais elevada entre os miscigenados, havendo diferença entre as duas categorias citadas $(p=0,048)$. Além disso, a medida FI-FPME foi menor entre os brancos e mais elevada entre os negros, ocorrendo diferenças entre os brancos com as outras duas categorias ancestrais $(p=0,023)$ (Tabela 4).

\section{DISCUSSÃO}

No Brasil, poucas são as pesquisas voltadas ao estudo antropológico forense na população ${ }^{5,15}$. Isso faz com que pesquisadores e profissionais forenses precisem remeter seu embasamento técnico e científico em estudos focados em populações estrangeiras, sobretudo europeias e asiáticas, o que, de fato, não representa, com fidelidade, a realidade métrica e morfológica de um grupo populacional tão miscigenado como o brasileiro.

Segundo Durso et al. $(2014)^{16}$, a população brasileira é uma das mais miscigenadas do mundo, sendo tal fato reflexo de quinhentos anos de cruzamentos interétnicos, sobretudo, de três raízes ancestrais: ameríndios (indígenas americanos), europeus e africanos. Ainda de acordo com esses pesquisadores, a proporção de contribuição de cada uma dessas ancestralidades varia de acordo com a região de interesse de estudo, sendo a extensão dessa mistura de tal forma que, muitas vezes, não é possível distinção fenotípica entre os indivíduos analisados.

No presente estudo, apenas duas variáveis apresentaram alguma associação entre as medidas do palato e as ancestralidades descritas para a amostra brasileira em questão, reforçando a questão da dificuldade

Tabela 1. Estatísticas das variáveis de interesse para o estudo do palato ósseo

\begin{tabular}{l|c|c|c|c}
\hline \multicolumn{1}{c|}{$\mathrm{n}=178$} & Média $\pm \mathrm{DP}$ & Mediana & Mínimo & Máximo \\
\hline FI-FPMD & $39,62 \pm 2,77$ & 39,70 & 29,66 & 47,27 \\
FI-FPME & $39,99 \pm 2,61$ & 40,11 & 32,51 & 47,87 \\
FPMD-FPME & $35,19 \pm 3,03$ & 35,17 & 26,16 & 44,99 \\
FI-ENP & $41,22 \pm 3,35$ & 41,40 & 30,46 & 50,47 \\
\hline
\end{tabular}


Tabela 2. Distribuição da amostra quanto ao sexo segundo as medições palatinas

\begin{tabular}{|c|c|c|c|}
\hline \multirow{3}{*}{$n=178$} & \multicolumn{2}{|c|}{ Sexo } & \multirow{3}{*}{ Valor de $p$} \\
\hline & Masculino & Feminino & \\
\hline & $\begin{array}{c}\text { Média } \pm \text { DP } \\
\text { (Mediana) }\end{array}$ & $\begin{array}{c}\text { Média } \pm \text { DP } \\
\text { (Mediana) }\end{array}$ & \\
\hline FI-FPMD & $\begin{array}{c}40,40 \pm 2,53 \\
(40,61)\end{array}$ & $\begin{array}{c}38,69 \pm 2,76 \\
(39,15)\end{array}$ & $\mathrm{p}^{(1)}<0,001^{*}$ \\
\hline FI-FPME & $\begin{array}{c}40,64 \pm 2,59 \\
(40,69)\end{array}$ & $\begin{array}{c}39,21 \pm 2,42 \\
(39,40)\end{array}$ & $\mathrm{p}^{(1)}<0,001^{*}$ \\
\hline FPMD-FPME & $\begin{array}{c}36,12 \pm 2,82 \\
(35,99)\end{array}$ & $\begin{array}{c}34,06 \pm 2,90 \\
(33,95)\end{array}$ & $\mathrm{p}^{(1)}<0,001^{*}$ \\
\hline FI-ENP & $\begin{array}{c}42,22 \pm 3,09 \\
(42,13)\end{array}$ & $\begin{array}{c}40,02 \pm 3,27 \\
(40,06)\end{array}$ & $\mathrm{p}^{(1)}<0,001^{*}$ \\
\hline
\end{tabular}

$\left({ }^{\star}\right)$ : Diferença significativa ao nível de $5,0 \%$.

(1): Através do teste t-Student com variâncias iguais.

Tabela 3. Distribuição da amostra quanto à faixa etária segundo as medições palatinas.

\begin{tabular}{|c|c|c|c|c|}
\hline \multirow{3}{*}{$n=178$} & \multicolumn{3}{|c|}{ Faixa etária } & \multirow{3}{*}{ Valor de $p$} \\
\hline & Até 39 & 40 a 59 & 60 ou mais & \\
\hline & $\begin{array}{c}\text { Média } \pm \text { DP } \\
\text { (Mediana) }\end{array}$ & $\begin{array}{c}\text { Média } \pm \text { DP } \\
\text { (Mediana) }\end{array}$ & $\begin{array}{c}\text { Média } \pm \text { DP } \\
\text { (Mediana) }\end{array}$ & \\
\hline FI-FPMD & $\begin{array}{c}39,34 \pm 2,88 \\
(39,29)\end{array}$ & $\begin{array}{c}39,92 \pm 2,51 \\
(40,24)\end{array}$ & $\begin{array}{c}39,53 \pm 2,90 \\
(39,68)\end{array}$ & $p^{(1)}=0,567$ \\
\hline FI-FPME & $\begin{array}{c}39,67 \pm 2,80 \\
(40,00)\end{array}$ & $\begin{array}{c}40,30 \pm 2,53 \\
(40,41)\end{array}$ & $\begin{array}{c}39,91 \pm 2,58 \\
(39,82)\end{array}$ & $p^{(1)}=0,485$ \\
\hline $\begin{array}{l}\text { FPMD- } \\
\text { FPME }\end{array}$ & $\begin{array}{c}34,19 \pm 3,40 \\
(34,24)\end{array}$ & $\begin{array}{c}35,52 \pm 2,96 \\
(35,62)\end{array}$ & $\begin{array}{c}35,41 \pm 2,83 \\
(35,24)\end{array}$ & $p^{(1)}=0,072$ \\
\hline FI-ENP & $\begin{array}{c}41,57 \pm 3,47 \\
(41,74)\end{array}$ & $\begin{array}{c}41,61 \pm 3,46 \\
(42,28)\end{array}$ & $\begin{array}{c}40,78 \pm 3,20 \\
(40,87)\end{array}$ & $p^{(1)}=0,269$ \\
\hline
\end{tabular}

(*): Diferença significativa ao nível de 5,0\%.

${ }^{(1)}$ : Através do teste F(ANOVA) com comparações pareadas de Tukey.

em se trabalhar com esse parâmetro na população brasileira.

Com relação à análise do dimorfismo sexual, lembra-se que as diferenças anatômicas entre os sexos masculino e feminino vão além dos caracteres físicos primários e secundários. Os esqueletos ósseos de homens e mulheres, apesar de possuírem o mesmo número de constituintes ósseos, carregam características morfológicas e métricas ${ }^{5}$ capazes de diferenciá-los quanto ao sexo, à ancestralidade e à idade. Dentre os 
Tabela 4. Distribuição da amostra quanto à ancestralidade segundo as medições palatinas

\begin{tabular}{|c|c|c|c|c|}
\hline \multirow{3}{*}{$n=178$} & \multicolumn{3}{|c|}{ Ancestralidade } & \multirow{3}{*}{ Valor de p } \\
\hline & Branco & Negro & Miscigenado & \\
\hline & $\begin{array}{l}\text { Média } \pm \text { DP } \\
\text { (Mediana) }\end{array}$ & $\begin{array}{c}\text { Média } \pm \text { DP } \\
\text { (Mediana) }\end{array}$ & $\begin{array}{c}\text { Média } \pm \text { DP } \\
\text { (Mediana) }\end{array}$ & \\
\hline FI-FPMD & $\begin{array}{c}39,19 \pm 2,83 \\
(39,41)^{(A)}\end{array}$ & $\begin{array}{c}40,13 \pm 2,53 \\
(40,30)^{(A B)}\end{array}$ & $\begin{array}{c}40,26 \pm 2,61 \\
(40,70)^{(B)}\end{array}$ & $\mathrm{p}^{(1)}=0,048^{*}$ \\
\hline FI-FPME & $\begin{array}{c}39,54 \pm 2,55 \\
(39,62)^{(A)}\end{array}$ & $\begin{array}{c}40,70 \pm 2,40 \\
(40,69)^{(B)}\end{array}$ & $\begin{array}{c}40,58 \pm 2,68 \\
(40,75)^{(B)}\end{array}$ & $\mathrm{p}^{(1)}=0,023^{*}$ \\
\hline FPMD-FPME & $\begin{array}{c}34,96 \pm 3,05 \\
(34,87)\end{array}$ & $\begin{array}{c}35,68 \pm 2,98 \\
(35,31)\end{array}$ & $\begin{array}{c}35,42 \pm 3,03 \\
(35,65)\end{array}$ & $\mathrm{p}^{(2)}=0,351$ \\
\hline FI-ENP & $\begin{array}{c}40,85 \pm 3,44 \\
(41,13)\end{array}$ & $\begin{array}{c}42,14 \pm 3,37 \\
(42,41)\end{array}$ & $\begin{array}{c}41,55 \pm 3,07 \\
(41,55)\end{array}$ & $p^{(1)}=0,163$ \\
\hline
\end{tabular}

$\left(^{*}\right)$ : Diferença significativa ao nível de $5,0 \%$.

(1): Através do teste F(ANOVA) com comparações pareadas de LSD.

$\left(^{2}\right)$ : Através do teste de Kruskal Wallis com comparações pareadas do referido teste.

NOTA: Se todas as letras entre parênteses são distintas, comprova-se diferença significativa entre as categorias correspondentes.

componentes anatômicos, o palato duro, que desempenha importantes funções no sistema estomatognático, é relatado como uma estrutura possível para a avaliação antropométrica de uma população ${ }^{5,17-19}$.

Estudo utilizando quatrocentos crânios humanos de indivíduos portugueses brancos, de 18 a 70 anos $^{19}$, provou que as medidas palatinas referentes à altura (FI-ENP) e à largura (FPMD-FPME) são maiores para o sexo masculino $(52,9 \mathrm{~mm}$ e $38,5 \mathrm{~mm}$, respectivamente) do que para o feminino $(51,0 \mathrm{~mm}$ e $37,4 \mathrm{~mm}$, respectivamente). Os resultados apresentados por este estudo corroboram com os achados da pesquisa atual, porém nesta, as métricas foram inferiores: o sexo masculino teve média de $42,22( \pm 3,09) \mathrm{mm}$ e $36,12( \pm 2,82) \mathrm{mm}$ para a altura e a largura, respectivamente, enquanto o feminino apresentou-se com 40,02 $( \pm 3,27)$ e $34,06( \pm 2,90)$ para as mesmas medidas.

Pesquisa realizada com 100 crânios secos humanos provenientes de um cemitério da região centro-oeste brasileira, com idades entre 22 e 55 anos $^{5}$, examinou as medidas FI-FPMD, FI-FPME e FPMD-FPME. Os autores relataram diferença estatisticamente diferente entre os sexos para as dimensões FI-FPMD e FI-FPME.

Jacob $(2016)^{17}$, por sua vez, estudando metricamente 90 crânios secos da região sul da Índia, observou altura média de $39,8 \mathrm{~mm}$ e $37,2 \mathrm{~mm}$ e largura média de $35,3 \mathrm{~mm}$ e $32,3 \mathrm{~mm}$ para os sexos masculino e feminino, respectivamente, sendo esses resultados estatisticamente dimórficos. Tais resultados mostram que as medidas de interesse para esta população indiana são inferiores às brasileiras, retificando o fato de que tabelas de referências de um grupo populacional não podem ser aludidas a outros ${ }^{20}$.

\section{CONCLUSÃO}

Medidas palatinas podem ser usadas como um método auxiliar na análise de crânios 
secos completos ou em casos em que restos fragmentados com palato ósseo intacto estão disponíveis para identificação forense. Para a população brasileira estudada, concluiu-se que as características do palato ósseo podem ser aplicadas como maior confiabilidade para estimativa do sexo.

\section{REFERÊNCIAS}

1. Almeida Júnior E, Reis FP, Galvão LCC, Rosa HRS, Costa N. Sex and age estimation by cranial measurements. Revista Bahiana de Odontologia. 2015; 6(2):81-88.

2. Silva JTSO. Antropologia Forense e Identificacao Humana [Dissertação]. Porto: Universidade Fernando Pessoa; 2015. 81p.

3. Lino-Júnior HL, Gabriel M, Daruge-Júnior E, Silva RHA. Ensino de Odontologia Legal no Brasil: um convite à reflexão. Revista da ABENO. 2015; 15(2):38-46.

4. Abe DM. Avaliação do sexo por análise de função discriminante a partir de dimensões lineares do crânio [Dissertação de Mestrado]. Piracicaba: Universidade Estadual de Campinas; 2000. 137p.

5. Lima LNC, Oliveira OF, Sassi C, Picapedra A, Francesquini Júnior L, Daruge Júnior $E$. Sex determination by linear measurements of palatal bones and skull base. JFOS, 2012; 30(1):37-44.

6. Madeira MC. Anatomia da face: Bases anatomo-funcionais para prática odontológica. $8^{\mathrm{a}}$. ed. São Paulo: Sarvier; 2013.

7. Silveira GF. Considerações anatómicas sobre o enveIhecimento do aparelho estomatognático [Dissertação de Mestrado]. Porto: Universidade Fernando Pessoa; 2016. 103p.

8. Kimmerle EH, Ross A, Slice D. Sexual dimorphism in America: geometric morphometric analysis of the craniofacial region. J. Forensic Sci. 2008; 53(1):54-57.

9. Sarilita E, Soames R. Morphology of the hard palate: a study of dry skulls and review of the literature. Rev Arg de Anat Clin. 2015; 7(1):34-43.

10. Dantas JSL, Almeida Júnior E, Galvão LCC. Estimation of Sex and Age through the Cranial Nasal Index. Brazilian Journal of Forensic Sciences, Medical Law and Bioethics. 2015; 4(4):419-429.

11. Almeida Júnior E, Reis FP, Galvão LCC, Alves MC, Vasconcelos D. Investigação do sexo e idade por meio de mensurações interforames em crânios secos de adultos. Rev. Ciênc. Méd. Biol. 2013; 12(1):55-59.

\section{AGRADECIMENTOS}

Este estudo foi financiado pela Coordenação de Aperfeiçoamento de Pessoal de Nível Superior - Brasil (CAPES) e pela Fundação de Amparo à Ciência e Tecnologia de Pernambuco (FACEPE).

12. França GV. Medicina Legal. 10ª . ed. Rio de Janeiro: Guanabara Koogan; 2017.

13. Vanrell JP. Odontologia Legal e Antropologia Forense. $2^{a}$. ed. Rio de Janeiro: Guanabara Koogan; 2019.

14. Veyre-Goulet SA, Mercier C, Robin O, Guerin C. Recent human sexual dimorphism study using cephalometric plots on lateral teleradiography and discriminant function analysis. J Forensic Sci. 2008; 53(4):786-789.

15. Gonçalves PC. Estudo de 25 crânios de indivíduos do Rio Grande do Sul: inferência de sexo e de ancestralidade com o uso de cranioscopia, craniometria e genética forense [Dissertação de Mestrado]. Porto Alegre: Pontifícia Universidade Católica do Rio Grande do Sul; 2014. 44p.

16. Durso DF, Bydlowski SP, Hutz MH, Suarez-Kurtz G, Magalhães TR, Pena SDJ. Association of Genetic Variants with Self-Assessed Color Categories in Brazilians. PLoS ONE. 2014; 9(1):e83926.

17. Jacob M, Bindhu S, Avadhani R. Sex determination from hard palate measurements using palatine index with reference to its clinical implications. Indian Journal of Clinical Anatomy and Physiology. 2016; 3(2):186-188.

18. Saliba CA. Contribuição ao estudo do dimorfismo sexual através de medidas do crânio [Tese de Doutorado]. Piracicaba: Universidade Estadual de Campinas; 1999. 132p.

19. Volkmann O, Cotrim-Ferreira FA, Villi ÉR, Ferreira-Tormin AC, Júnior HS, Vellini-Ferreira F. Estudo Antropométrico das Correlações de Medidas Lineares do Palato, do Crânio e da Face. Ortodon Ortop Facial. 2003; 46(8):307-314.

20. Nunes FB, Gonçalves PC. A importância da craniometria na criminalística: revisão de literatura. Rev. Bras. Crimin. $2014 ; 3(1): 36-43$.

\section{CORRESPONDÊNCIA}

Larissa Chaves Cardoso Fernandes

Avenida Presidente Epitácio Pessoa, 4050,

Apartamento 1701 - Miramar

João Pessoa - Paraíba, Brasil. CEP: 58032-000.

E-mail: larissaccfernandes@gmail.com 